\title{
CONTROLE DA PODRIDÃO PARDA DO PESSEGUEIRO COM FUNGICIDAS E FOSFITOS AVALIADOS EM PRÉ E PÓS-COLHEITA
}

\author{
Control of peach tree brown rot by fungicides and phosphites \\ evaluated during preharvest and postharvest
}

\author{
Luciene Martins Moreira ${ }^{1}$, Louise Larissa May-de Mio
}

\begin{abstract}
RESUMO
A podridão parda é a doença mais importante para a cultura do pessegueiro, entretanto, no Brasil são escassos os trabalhos realizados a campo visando o seu controle. Objetivou-se, neste trabalho, selecionar fungicidas em laboratório e avaliar a sua eficiência e de fosfitos a campo, para o controle da podridão parda monitorando as fases de desenvolvimento de frutos e pós-colheita, além de avaliar as características qualitativas dos frutos. O experimento de campo foi realizado com seis tratamentos e quatro repetições: três fungicidas pré-selecionados in vitro (iminoctadine tris albesilate, myclobutanil e iprodione), dois fosfitos (CaB e de $\mathrm{K}$ ) e testemunha. Foi avaliada a incidência de infecções latentes de Monilinia fructicola em frutos em desenvolvimento e em frutos maduros após a colheita. Para os frutos em desenvolvimento observou-se maior incidência nas duas últimas coletas. No campo, o iprodione e o iminoctadine mostraram eficiência no controle da doença durante as avaliações. Após três dias no ambiente o iminoctadine foi melhor que os demais tratamentos mantendo a incidência da podridão parda em 1,0\% contra 31,4\% no tratamento com iprodione e $91,2 \%$ na testemunha. $\mathrm{O}$ fosfito de $\mathrm{CaB}$ não mostrou diferença em relação à testemunha no decorrer das avaliações, mas o fosfito de $\mathrm{K}$, reduziu em 60 e $28 \%$ o número de frutos doentes aos três e cinco dias, respectivamente, em relação à testemunha. Quanto aos parâmetros de qualidade, o peso médio dos frutos, o diâmetro e a firmeza da polpa, não mostraram diferenças significativas em relação à testemunha.
\end{abstract}

Termos para indexação: Monilinia fructicola, pêssego, controle químico, infecção latente.

\begin{abstract}
Brown rot is the most important disease in peach tree cultivation, but field studies with control methods are currently rare in Brazil. One of the objectives of this study was to select fungicides in the laboratory then test them in the field, additionally to phosphites, for the control of the brown rot. The control was performed by observing the fruit development phase and by postharvest monitoring. Another objective was to assess the qualitative characteristics of the fruit. The field experiment was carried out with six treatments and four replications: three in vitro pre-selected fungicides (iminoctadine tris albesilate, myclobutanil and iprodione), two phosphites (CaB and $\mathrm{K}$ ) and the control. Latent infections in developing and postharvest fruits were assessed in regard to the incidence of Monilinia fructicola. For the developing fruits it was observed higher incidence during the two last assessments. In the field, iprodione and iminoctadine showed efficient control of the disease during the assessments. After three days in the environment, the iminoctadine was better than the other treatments, keeping the incidence of brown rot to $1.02 \%$ as against $31.4 \%$ for iprodione and $91.2 \%$ for the control. Phosphite- $\mathrm{CaB}$ showed no difference in relation to the control as the assessments proceeded, but phosphate-K reduced the number of diseased fruits over three and five days by $60 \%$ and $28 \%$, respectively, in relation to the control. In regard to quality parameters, there was no significant difference in the average fruit weight, diameter and pulp firmness in relation to the control.
\end{abstract}

Index terms: Monilinia fructicola, peach, chemical control, latent infection.

(Recebido em 23 de maio de 2007 e aprovado em 11 de setembro de 2008)

\section{INTRODUÇÃO}

A podridão parda causada pelo fungo Monilinia fructicola (Wint Honey) é a doença mais importante das rosáceas de caroço. O método de controle mais adotado é o químico, com pulverizações de fungicidas desde a floração à pré-colheita (MAY-DE MIO et al., 2004a; MOREIRA, 2005), sendo os produtos iprodione, triforine, procimidone, captana, mancozebe recomendados para o controle da doença (MAY-DE MIO et al., 2004b). Apesar da eficiência de muitos produtos específicos o uso repetido de ingredientes ativos pode levar ao desenvolvimento de resistência do patógeno, sendo ideal para o manejo da doença a adoção de fungicidas com diferentes mecanismos de ação (EMERY et al., 2002).

Uma alternativa para o manejo é o controle com novos produtos, como por exemplo, os fosfitos, cuja ação é a indução à formação de fitoalexinas, como já relatado em outras culturas como macieira e videira (BONETI \&

\footnotetext{
'Engenheira Agrônoma - Departamento de Fitotecnia e Fitossanitarismo/DFF - Universidade Federal do Paraná/UFPR - Rua dos Funcionários, 1540 80.035-050 - Curitiba, PR - Immoreira@terra.com.br

Engenheira Agrônoma, Doutora em Fitopatologia - Departamento de Fitotecnia e Fitossanitarismo/DFF - Universidade Federal do Paraná/UFPR - Rua dos Funcionários, 1540 -80.035-050 - Curitiba, PR - maydemio@ufpr.br
} 
KATSURAYAMA, 2002; DERCKS \& CREASY, 1989, citados por SÔNEGO et al., 2003). Além de alternativas para o manejo da doença, é fundamental o acompanhamento da eficiência dos tratamentos durante o ciclo, pois o fungo $M$. fructicola pode afetar as flores, permanecer latente nos frutos em desenvolvimento, ou desenvolver sintomas durante a colheita, armazenagem e comercialização (BYRDE \& WILLITS, 1977; MAY-DE MIO et al., 2004a).

Muitos trabalhos têm sido realizados visando o controle da doença em pré-colheita (FORTES, 1994; MEDEIROS \& MEDEIROS 1997a; WADT et al., 1999), mas as avaliações normalmente consideram apenas a porcentagem de podridão parda na colheita. Faltam estudos da eficiência de fungicidas e dos fosfitos a campo relacionando a ocorrência da doença na fase de frutos em desenvolvimento e em pós-colheita e também informações sobre a interferência dos tratamentos realizados a campo nas características qualitativas do fruto.

Objetivou-se, neste trabalho: i) selecionar, in vitro, fungicidas para o controle do desenvolvimento do fungo M. fructicola; ii) avaliar a eficiência de fungicidas e fosfitos no campo para o controle da podridão parda monitorando as fases de desenvolvimento de frutos (infecção latente) e pós-colheita; iii) verificar relação da podridão parda latente em pré-colheita com a doença que se desenvolve após a colheita, durante a comercialização; e, iv) avaliar o efeito dos tratamentos com fungicidas e fosfitos nas características qualitativas (peso, firmeza de polpa, diâmetro, sólido solúveis) dos frutos de pessegueiro.

\section{MATERIAL E MÉTODOS}

Seleção de fungicidas in vitro para o controle químico de M. fructicola

Foram testados in vitro os seguintes ingredientes ativos de fungicidas: benomyl, captana, carbendazim, imibenconazole, iminoctadine tris albesilate, iprodione, mancozebe, myclobutanil, procimidone, thiram, tiofanato metílico, triforine, vinclozolin. Os fungicidas foram incorporados individualmente ao meio de cultura batatadextrose-ágar (BDA) nas concentrações de $100 \mathrm{mg} \mathrm{L}^{-1}, 10$ $\mathrm{mg} \mathrm{L}^{-1}, 1,0 \mathrm{mg} \mathrm{L}^{-1}$ e $0,1 \mathrm{mg} \mathrm{L}^{-1}$. Discos de micélio $(0,5 \mathrm{~cm}$ de diâmetro) de $M$. fructicola (isolado UFPR-IM3) foram repicados em presença e em ausência (testemunha) dos fungicidas citados. Para a avaliação, mediu-se o diâmetro da colônia, a cada 24 horas, até que a testemunha atingisse dois terços da placa. A análise dos resultados foi feita para delineamento inteiramente casualizado, com arranjo fatorial de 13 (fungicidas) x 4 (concentrações), com cinco repetições pelo programa MSTAT $®$.

\section{Avaliação da eficiência de fungicidas e fosfitos no controle da podridão parda a campo}

O experimento foi implantado no município da Lapa (Paraná) e a área experimental foi de um hectare, com plantas de quatro anos de idade, cultivar BR-1, de ciclo tardio, espaçadas em seis metros nas entrelinhas e três metros entre plantas. $\mathrm{O}$ experimento teve delineamento em blocos casualizados com quatro repetições e seis tratamentos, com unidade experimental de nove plantas, totalizando 216 plantas. Três tratamentos foram realizados com os fungicidas selecionados in vitro, iminoctadine tris albesilate (100 mL p.c.- produto comercial), myclobutanil (12 g p.c.), e iprodione (150 mL p.c.), aplicados para $100 \mathrm{~L}$ de água (considerando uma calda de 1000 L/ha). Dois tratamentos foram realizados com os fosfitos de $\mathrm{CaB}$ e de $\mathrm{K}$ (200 mL para $100 \mathrm{~L}$ de água) e todos foram comparados com a testemunha, sem controle. Os tratamentos foram aplicados em pré-colheita, em cinco pulverizações com intervalo de 10 dias, iniciando-se as pulverizações em final do mês de outubro, após o raleio.

Para monitoramento das infecções latentes, em frutos em desenvolvimento, foram feitas cinco coletas de 24 frutos/tratamento, sendo seis frutos/parcela, antes de cada pulverização. Conforme metodologia adaptada de Northover \& Cerkauskas (1994) os frutos vindos do campo foram imersos em solução de etanol (70\%) e depois em hipoclorito de sódio (2\%), por dois minutos em cada solução. Os frutos foram posteriormente lavados em água esterilizada e postos em potes plásticos individualizados contendo papel-filtro umedecido. Após 10 dias, os frutos foram avaliados quanto à incidência de podridão parda.

Para a avaliação da incidência da doença em póscolheita foram coletados 50 frutos por parcela da planta central. Em seguida, os frutos foram armazenados em câmara fria por um período de seis dias a $5^{\circ} \mathrm{C}$, sendo então retirados e colocados em bancadas, permanecendo à temperatura ambiente (cerca de $25{ }^{\circ} \mathrm{C}$ ), visando simular condições de mercado. Foram realizadas três avaliações de incidência da doença: no momento da retirada da câmara fria, no terceiro e no quinto dia de manutenção no ambiente, após refrigeração.

Como variáveis relacionadas à qualidade do fruto foram avaliadas: peso médio de frutos, diâmetro médio de frutos, sólidos solúveis (brix) e firmeza da polpa, considerando uma amostra de seis frutos/parcela, avaliados logo após a colheita e também coletados da planta central. A análise dos resultados foi feita para delineamento em blocos casualisados, com seis tratamentos e quatro 
repetições, sendo utilizada quando necessária à transformação arcoseno $\sqrt{\mathrm{x}}$ para os testes de comparação de média (DMS), pelo programa MSTATß.

\section{RESULTADOS E DISCUSSÃO}

\section{Efeito de fungicidas in vitro para o controle de $M$.} fructicola

Na concentração de $100 \mathrm{mg} \mathrm{L}^{-1}$, todos os produtos inibiram o crescimento micelial de $M$. fructicola e vários impediram totalmente o crescimento do patógeno (benomyl, carbendazim, iminoctadine tris albesilate, iprodione, mancozebe, myclobutanil, procimidone, tiofanato metílico e vinclozolin) (Tabela 1). Com a diminuição da concentração para $10 \mathrm{mg} \mathrm{L}^{-1}$, o mancozebe e o thiram tiveram suas eficiências reduzidas; captana, tiofanato metílico e triforine foram intermediários e os demais continuaram eficientes. Com a concentração de 1,0 $\mathrm{mg} \mathrm{L}^{-1}$ destacou-se o procimidone com $100 \%$ de inibição do crescimento do patógeno, seguido por imibenconazole e iprodione inibindo acima de $70 \%$ (Tabela 1). Em concordância com os dados deste trabalho, Penrose et al. (1985) relataram a capacidade inibitória do benomyl, vinclozolin, iprodione e procimidone ao crescimento micelial de M. fructicola e, Ritchie (1982) evidenciou a toxicidade inerente de vinclozolin e iprodione que inibiram $100 \%$ do crescimento micelial na concentração de $25 \mathrm{mg} \mathrm{L}^{-1}$.
A $0,1 \mathrm{mg} \mathrm{L}^{-1}$ a maior inibição foi obtida com o vinclozolin. O melhor efeito dos fungicidas a $1,0 \mathrm{mg} \mathrm{L}^{-1}$ e $0,1 \mathrm{mg} \mathrm{L}^{-1}$ é devido a esses produtos serem mais específicos para o grupo de fungos.

\section{Eficiência de fungicidas e fosfitos aplicados a campo no controle da podridão parda}

Foram observados altos porcentuais de infecções latentes em frutos na pré-colheita, principalmente nas duas últimas coletas (Figura 1). Tais infecções tiveram relação direta com o que ocorreu durante a pós-colheita, entretanto apenas a última avaliação no campo (4 dias antes da colheita) indica um alto coeficiente de determinação $\left(\mathrm{R}^{2}=0,90\right)$, mas quando considerou-se a penúltima avaliação (14 dias antes da colheita), o coeficiente de determinação foi baixo $\left(\mathrm{R}^{2}=0,36\right)$ (Figura $2 \mathrm{~A}$ e $2 \mathrm{~B}$, respectivamente). A diagnose de infecções latentes em pré-colheita pode auxiliar o produtor a adequar seu planejamento de controle da doença, durante o processo de comercialização, no entanto para uma maior segurança a coleta dos frutos deve ser feita próxima da data de colheita. Os tratamentostestemunha e fosfito de $\mathrm{CaB}$ tiveram a maior porcentagem de podridão parda e o iminoctadine apresentou a menor porcentagem de doença (Figura 1). Os produtos que indicaram melhor controle foram o iminoctadine e o fosfito de $\mathrm{K}$, não apresentando sintomas da doença até a penúltima

Tabela 1 - Porcentagem de inibição do crescimento micelial de colônias de um isolado de Monilinia fructicola em relação à testemunha, em diferentes concentrações de fungicidas.

\begin{tabular}{lrrrr}
\hline \multirow{2}{*}{ Ingredientes ativos } & \multicolumn{5}{c}{ Inibição $(\%)$ nas concentrações $\left(\mathrm{mg} \mathrm{L}^{-1}\right)^{1,2}$} \\
\cline { 2 - 5 } & 100 & 10 & 1,0 & 0,1 \\
\hline Benomyl & $100,00 \mathrm{~ns}$ & $100,00 \mathrm{a}$ & $7,82 \mathrm{~h}$ & $1,71 \mathrm{f}$ \\
Captana & 90,83 & $26,36 \mathrm{~b}$ & $12,00 \mathrm{gh}$ & $0,0 \mathrm{f}$ \\
Carbendazim & 100,00 & $100,00 \mathrm{a}$ & $26,87 \mathrm{fg}$ & 12,26 ef \\
Imibenconazole & 88,33 & $85,10 \mathrm{a}$ & $79,81 \mathrm{~b}$ & 43,29 abc \\
Iminoctadine tris albesilate & 100,00 & $95,90 \mathrm{a}$ & $62,74 \mathrm{~cd}$ & $46,11 \mathrm{ab}$ \\
Iprodione & 100,00 & $92,19 \mathrm{a}$ & $71,94 \mathrm{bc}$ & $36,05 \mathrm{bcd}$ \\
Mancozebe & 100,00 & $7,84 \mathrm{c}$ & $17,84 \mathrm{gh}$ & $7,69 \mathrm{f}$ \\
Myclobutanil & 100,00 & $100,00 \mathrm{a}$ & $48,38 \mathrm{de}$ & $32,53 \mathrm{bcd}$ \\
Procimidone & 100,00 & $100,00 \mathrm{a}$ & $100,00 \mathrm{a}$ & $25,89 \mathrm{de}$ \\
Thiram & 96,00 & $4,42 \mathrm{c}$ & $13,52 \mathrm{gh}$ & 14,62 ef \\
Tiofanato metílico & 100,00 & $42,49 \mathrm{~b}$ & $38,79 \mathrm{ef}$ & $27,36 \mathrm{cde}$ \\
Triforine & 88,70 & $41,29 \mathrm{~b}$ & $18,19 \mathrm{gh}$ & 12,67 ef \\
Vinclozolin & 100,00 & $100,00 \mathrm{a}$ & $41,11 \mathrm{ef}$ & $53,12 \mathrm{a}$ \\
\hline
\end{tabular}

${ }^{1}$ Dados originais. As médias seguidas verticalmente pela mesma letra não diferem estatisticamente pelo DMS teste, a $1 \%$ de significância. Coeficiente Variação: 17,6 \%.

${ }^{2}$ Porcentagem obtida por meio da medida do diâmetro das colônias do patógeno a cada 24 horas. ns - não significativo. 
avaliação. Na avaliação final além desses, o myclobutanil também se destacou (Figura 1). A eficiência dos fungicidas do grupo das dicarboximidas já era esperada, entretanto, é interessante ressaltar a possibilidade do uso de um ingrediente ativo de grupo químico diferente (iminoctadine) e principalmente o uso do fosfito de $\mathrm{K}$, que teve eficiência equivalente ao padrão químico mais usado na região de estudo, o iprodione.

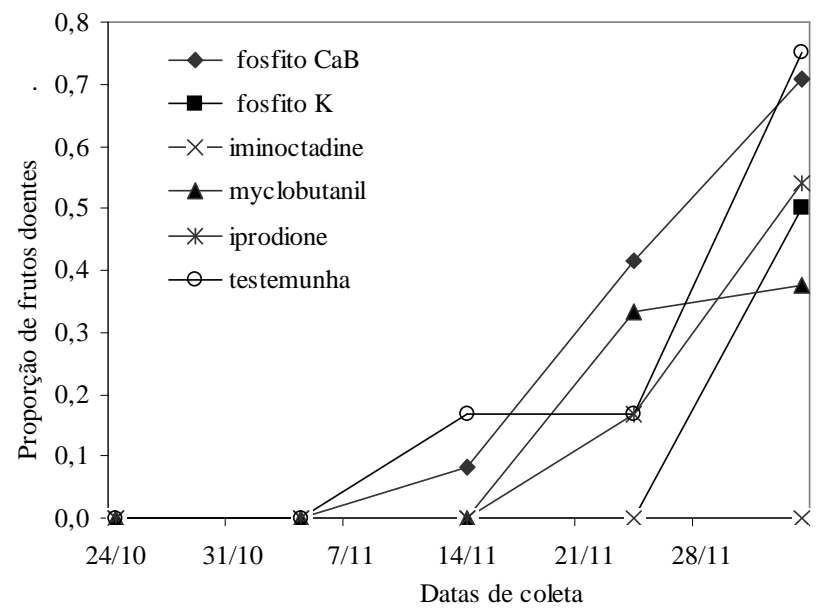

Figura 1 - Proporção de frutos, com infecção latente de Monilinia fructicola, determinada pelo método de imersão em etanol $70 \%$ e hipoclorito $2 \%$, durante a fase de crescimento de frutos, de pessegueiro, cultivar BR-1, sob diferentes tratamentos realizados entre o final da floração e a colheita. Lapa-PR.

Na avaliação feita em pós-colheita, imediatamente após a refrigeração a testemunha apresentava $12,3 \%$ de doença enquanto os tratamentos iminoctadine e iprodione tinham 0 e $1,5 \%$ de frutos com sintomas, respectivamente. Após três dias no ambiente (depois da refrigeração), observou-se um aumento da doença na testemunha para 91,2\% não diferindo apenas do fosfito de $\mathrm{CaB}$. Nessa avaliação, a menor incidência de podridão foi no tratamento com o fungicida iminoctadine, seguidos do iprodione, myclobutanil e fosfito de $K$, com redução de aproximadamente $50 \%$ da incidência da doença nos frutos. Aos cinco dias no ambiente $\left(25{ }^{\circ} \mathrm{C}\right)$, o iminoctadine continuou sendo o melhor tratamento atingindo $96 \%$ de controle e os tratamentos iprodione, myclobutanil e fosfito de $\mathrm{K}$ mantiveram diferença significativa da testemunha e do fosfito de $\mathrm{CaB}$ com 43, 29 e $28 \%$ de frutos sadios respectivamente, em relação à testemunha. Na primeira avaliação, a refrigeração ajudou a atrasar a exibição dos sintomas em frutos já contaminados. Porém, quando esses
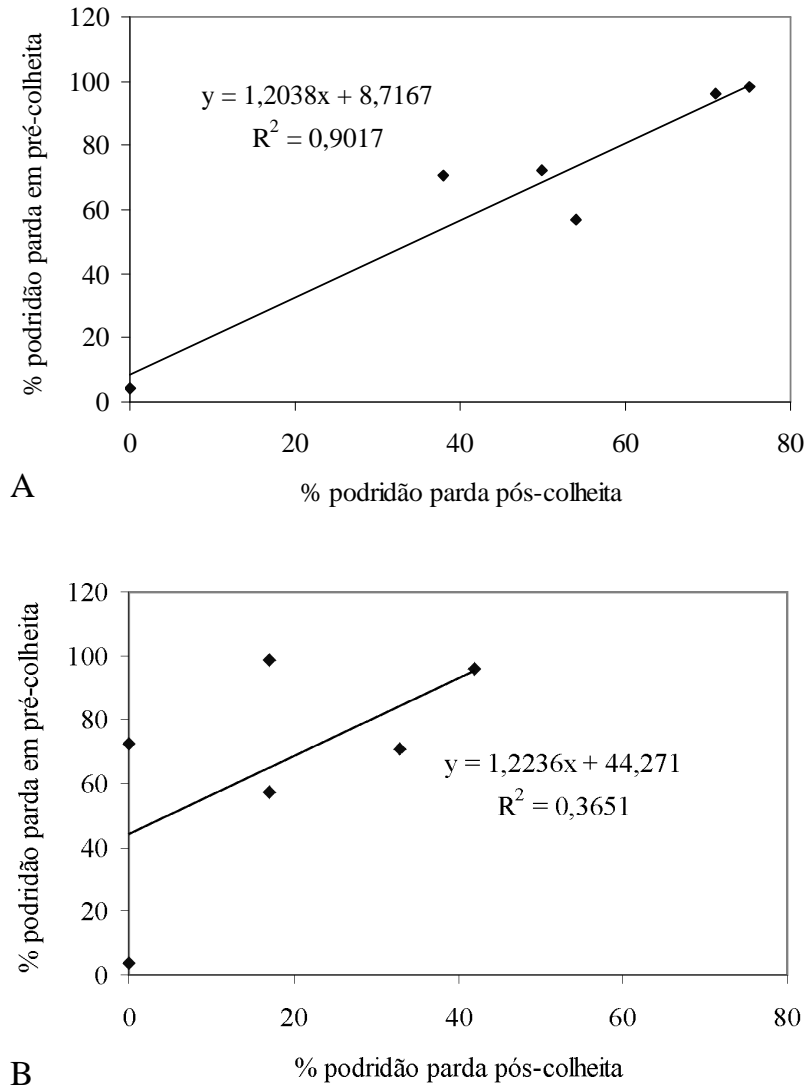

Figura 2 - Incidência de podridão parda latente em frutos de pessegueiro, cultivar BR-1, na pré-colheita, considerando-se a última avaliação (4 dias antes da colheita) (A) e, a penúltima avaliação (14 dias antes da colheita) (B) em relação à incidência da doença em pós-colheita (5 dias no ambiente após refrigeração).

foram expostos ao ambiente, a temperatura elevada auxiliou na expressão das infecções latentes antes contidas pela baixa temperatura e, o aumento do período de exposição resultou numa maior incidência de podridão parda, conforme o observado na última avaliação (Tabela 2).

Outra observação foi que, na ocasião da coleta dos frutos para a análise de podridão parda após a colheita, foram encontradas nas plantas-testemunha 18 frutos mumificados pelo patógeno e esse número foi reduzido em $23 \%$ no tratamento com fosfito de $\mathrm{CaB}, 56 \%$ com o iprodione e com o fosfito de $\mathrm{K} \mathrm{e}$, em torno de $80 \%$ com iminoctadine e myclobutanil (dados não apresentados).

$\mathrm{O}$ iprodione tem sido relatado como eficiente quando aplicado no campo, reduzindo a doença em póscolheita e diminuindo o diâmetro das lesões na superfície 
dos frutos (ANDRADE \& MATOS, 1996; BERTON et al., 1992; OSORIO et al., 1993). Além desses resultados, o iprodione e o iminoctadine controlaram a podridão parda quando utilizados em pomares de pessegueiro, no Rio Grande do Sul (FORTES, 1994; MEDEIROS \& MEDEIROS, 1997a,b). No caso de Medeiros \& Medeiros (1997a,b) as avaliações da incidência da doença foram aos três e cinco dias após a colheita, coincidindo com a metodologia desse trabalho, já Fortes (1994) avaliou somente aos três dias. O que diferencia o presente trabalho dos citados é a época de pulverização, a qual foi iniciada em pós-floração, garantindo assim um maior efeito dos tratamentos sobre as infecções latentes.

Tabela 2 - Incidência de podridão parda (\%) em pêssegos, cultivar BR-1, tratados em pré-colheita, com diferentes produtos e avaliados em três épocas, após seis dias de refrigeração e aos 0,3 e 5 dias no ambiente.

\begin{tabular}{|c|c|c|c|}
\hline Tratamentos & $\begin{array}{c}\text { Após } \\
\text { refrigeração } \\
(0 \text { dia })\end{array}$ & $\begin{array}{c}3 \text { dias } \\
\text { ambiente }\end{array}$ & $\begin{array}{c}5 \text { dias } \\
\text { ambiente }\end{array}$ \\
\hline Fosfito $\mathrm{CaB}$ & $8,52^{1} \quad a b$ & $70,69 \mathrm{ab}$ & 96,22 a \\
\hline Fosfito $\mathrm{K}$ & $4,64 \mathrm{ab}$ & 41,01 bc & $72,34 \mathrm{~b}$ \\
\hline $\begin{array}{l}\text { Iminoctadine } \\
\text { tris albesilate }\end{array}$ & $0,00 \mathrm{~b}$ & $1,02 \mathrm{~d}$ & 4,04 \\
\hline Myclobutanil & $3,51 \mathrm{ab}$ & $40,72 \mathrm{bc}$ & $70,86 \mathrm{~b}$ \\
\hline Iprodione & $1,52 \mathrm{~b}$ & $31,42 \mathrm{c}$ & $57,06 \mathrm{~b}$ \\
\hline Testemunha & $12,31 \mathrm{a}$ & $91,25 \mathrm{a}$ & 98,48 a \\
\hline $\begin{array}{l}\text { Coeficiente } \\
\text { Variação (\%) }\end{array}$ & 36,35 & 5,9 & 15,8 \\
\hline
\end{tabular}

${ }^{1}$ Dados originais. As médias seguidas verticalmente pela mesma letra não diferem estatisticamente pelo DMS teste, a $1 \%$ de significância.
Com o uso dos fosfitos de $\mathrm{CaB}$ e de $\mathrm{K}$ buscaramse alternativas aos fungicidas, pois entre os disponíveis, alguns não apresentam mais a eficiência desejada, além disso, podem ser úteis dentro de um manejo integrado da doença. A utilização dos fosfitos poderia ainda ser justificada por pesquisas que demonstraram que os nutrientes podem atuar como indutores de mecanismos de defesa e consequentemente melhorar a resistência das plantas às doenças (BARRETO \& CASTELLANI, 1994).

Na ocasião do experimento, não foram observados sintomas de fitotoxicidade nos tratamentos com fungicidas, porém, na fase final do trabalho, notou-se amarelecimento e queda de folhas das plantas tratadas com o fosfito de K. Portanto, coletaram-se amostras de folhas das plantas de todos os tratamentos, às quais passaram por análise química foliar para macro e micronutrientes. Os resultados indicaram níveis normais de nutrientes para todos os tratamentos, considerando-se que o material analisado foi colhido próximo ao final do ciclo da cultura quando os níveis de nutrientes podem apresentar-se abaixo dos padrões. Uma hipótese para a ocorrência do amarelecimento seria de que o tratamento com fosfito de $\mathrm{K}$ possa ter antecipado a senescência das folhas, porém, não interferiu negativamente na formação e desenvolvimento dos frutos desse ciclo, no peso, diâmetro, firmeza da polpa e sólidos solúveis (brix) (Tabela 3).

Os valores obtidos com a avaliação da firmeza da polpa para a cultivar BR-1, coincidem com os relatados para cultivares tardias de pessegueiro (KADER \& MITCHELL, 1989, citados por ARGENTA et al., 2004). Para sólidos solúveis apenas houve diferença estatística entre o fosfito de $\mathrm{K}$ e o iminoctadine em relação à testemunha. Entretanto, esses valores estão compatíveis aos

Tabela 3 - Sólidos solúveis, peso médio, diâmetro e firmeza da polpa em frutos de pessegueiro cultivar BR-1, sob diferentes tratamentos para controle de podridão parda em pré-colheita.

\begin{tabular}{lcccc}
\multicolumn{1}{c}{ Tratamentos } & $\begin{array}{c}\text { Sólidos } \\
\text { solúveis }^{1}\end{array}$ & $\begin{array}{c}\text { Peso médio } \\
(\mathrm{g})^{1}\end{array}$ & $\begin{array}{c}\text { Diâmetro } \\
(\mathrm{cm})^{1}\end{array}$ & $\begin{array}{c}\text { Firmeza } \\
(\mathrm{lb})^{1}\end{array}$ \\
\hline Fosfito CaB & $16,43 \mathrm{ab}$ & $78,66 \mathrm{~ns}$ & $5,51 \mathrm{~ns}$ & $13,50 \mathrm{~ns}$ \\
Fosfito K & $16,00 \mathrm{~b}$ & 82,84 & 5,24 & 15,08 \\
Iminoctadine tris albesilate & $15,86 \mathrm{~b}$ & 80,95 & 5,50 & 13,46 \\
Myclobutanil & $16,76 \mathrm{a}$ & 77,25 & 5,15 & 13,75 \\
Iprodione & $16,47 \mathrm{ab}$ & 81,01 & 5,25 & 14,46 \\
Testemunha & $16,96 \mathrm{a}$ & 79,36 & 5,36 & 14,25 \\
\hline Coeficiente Variação $(\%)$ & 2,19 & 8,65 & 4,01 & 6,57
\end{tabular}

${ }^{1}$ Dados originais. As médias seguidas verticalmente pela mesma letra não diferem estatisticamente pelo DMS teste, a $1 \%$ de significância. ns : não significativo. 
encontrados por Cantillano et al. (2003), em trabalho com cultivares de pêssego.

Nas condições desse experimento não foi encontrada diferença em relação ao tamanho e a coloração dos frutos das parcelas experimentais. Porém, há relatos da ocorrência de correlação positiva entre a nutrição potássica e o aumento da cor vermelha e do tamanho dos frutos. Além disso, a aplicação de $\mathrm{Ca}$ pode diminuir problemas com rachaduras nos frutos $\mathrm{e}$, juntamente com o $\mathrm{K}$, proporcionar uma textura mais firme aos pêssegos (PEREIRA et al., 1994).

Além da melhoria das características dos frutos já citadas, Biggs et al. (1997) relataram que o Ca possivelmente estimula a síntese de fitoalexinas, substâncias de defesa da planta contra o ataque do patógeno, e levantam a hipótese de que o nutriente passa agir diretamente sobre o patógeno, causando redução na virulência ou fungistase. Portanto, seria recomendado para um próximo estudo, que se prolongasse o período de pulverizações com os fosfitos, iniciando-se na floração até a pós-colheita, para verificar se haveria diferenças quanto à sua eficiência no controle da podridão parda.

\section{CONCLUSÕES}

Os melhores fungicidas da seleção in vitro foram procimidone, iminoctadine tris albesilate, iprodione, myclobutanil e o imibenconazole.

A pulverização do fosfito de $\mathrm{K}$, em pré-colheita reduziu a podridão em $26,5 \%$ e o fosfito de $\mathrm{CaB}$ não foi eficaz sob as mesmas condições.

$\mathrm{O}$ fungicida iminoctadine tris albesillate foi o produto de melhor desempenho no controle da podridão avaliada em pré e pós-colheita.

O peso médio dos frutos, o diâmetro e a firmeza da polpa não diferiram entre os tratamentos.

\section{REFERÊNCIAS BIBLIOGRÁFICAS}

ANDRADE, E. R.; MATOS, C. S. Controle químico de Monilinia fructicola em pêssego na pós-colheita.

Fitopatologia Brasileira, Brasília, v. 21, n. 2, p. 301-303, 1996.

ARGENTA, L. C.; CANTILLANO, F. F.; BECKER, W. F. Tecnologias pós-colheita para fruteira de caroço. In: MONTEIRO, L. B.; MAY-DE MIO, L. L.; SERRAT, B. M.; MOTTA, A. C.; CUQUEL, F. L. Fruteiras de caroço: uma visão ecológica. Curitiba: UFPR, 2004. p. 333-362.
BARRETO, M.; CASTELLANI, P. D. Relações entre a nutrição mineral e a incidência de doenças. In: SÁ, M. E. de; BUZZETI, S. Importância da adubação na qualidade dos produtos agrícolas. São Paulo: Ícone, 1994. p. 45-51.

BERTON, O.; SCHROEDER, A. L.; BLEICHER, J. Controle de podridões em pêssegos através de tratamentos em pré e pós-colheita. Agropecuária Catarinense, Santa Catarina, v. 5, n. 3, p. 4-5, 1992.

BIGGS, A. R.; EL-KHOLI, M. M.; EL-NESHAWY, S.; NICKERSON, R. Effects of calcium salts on growth, polygalacturonase activity, and infection of peach fruit by Monilinia fructicola. Plant Disease, Saint Paul, v. 81, n. 4, p. 399-403, 1997.

BONETI, J. I.; KATSURAYAMA, Y. Viabilidade do uso de fosfitos no manejo das doenças da macieira. In: ENCONTRO NACIONAL SOBRE FRUTICULTURA DE CLIMA TEMPERADO - ENFRUTE, 5., 2002, Fraiburgo. Anais... Fraiburgo, 2002. p. 125-139.

BYRDE, R. J. W.; WILLETTS, H. J. Infection. In: The brown rot of fruit: their biology and control. Oxford: Pergamon, 1977. cap. 7, p. 87-110.

CANTILLANO, F. F.; MADAIL, J. C. M.; MARTINS, C. R.; LAGOS, L. L.; SALVADOR, M. E.; FORTES, J. F.; REICHERT, L. J. Pêssego pós-colheita. Brasília, DF: Embrapa Informação Tecnológica, 2003. 47 p. (Frutas do Brasil, 51).

EMERY, K. M.; SCHERM, H.; SAVELLE, A. T.

Assessment of interactions between components of fungicide mixtures against Monilinia fructicola. Crop Protection, Oxford, v. 21, p. 41-47, 2002.

FORTES, J. F. Controle de Monilinia fructicola em Prunus persica na pré-colheita. Fitopatologia Brasileira, Brasília, v. 19, p. 327, 1994. Suplemento.

MAY-DE MIO, L. L.; GARRIDO, L.; UENO, B. Doenças de fruteiras de caroço. In: MONTEIRO, L. B.; MAY-DE MIO, L. L.; SERRAT, B. M.; MOTTA, A. C.; CUQUEL, F. L. Fruteiras de caroço: uma visão ecológica. Curitiba: UFPR, 2004a. p. 169-221.

MAY-DE MIO, L. L.; MONTEIRO, L. B.; NAZARENO, N. R. X. de; HICKEL, E. Classificação e manejo dos agroquímicos em fruteiras de caroço. In: MONTEIRO, L. 
B.; MAY-DE MIO, L. L.; SERRAT, B. M.; MOTTA, A. C.; CUQUEL, F. L. Fruteiras de caroço: uma visão ecológica. Curitiba: UFPR, 2004b. p. 263-297.

MEDEIROS, G.; MEDEIROS, C. A. Controle químico de Monilinia fructicola, em pessegueiro. Fitopatologia Brasileira, Brasília, v. 22, p. 283, 1997a. Suplemento.

MEDEIROS, G.; MEDEIROS, C. A. Efeito de fungicidas no controle de Monilinia fructicola em pessegueiro. Fitopatologia Brasileira, Brasília, v. 22, p. 283, 1997 b. Suplemento.

MOREIRA, L. M. Alternativas de controle integrado da podridão parda do pessegueiro. 2005. $113 \mathrm{f}$. Tese (Doutorado em Produção Vegetal) - Universidade Federal do Paraná, Curitiba, 2005.

NORTHOVER, J.; CERKAUSKAS, R. F. Detection and significance of symptomless latent infections of Monilinia fructicola in plums. Canadian Journal of Plant Pathology, Ottawa, v. 16, n. 1, p. 30-36, 1994.

OSORIO, J. M.; ADASKAVEG, J. E.; OGAWA, J.

Comparative efficacy and systemic activity of iprodione and the experimental anilide E-0858 for control of brown rot on peach fruit. Plant Disease, Saint Paul, v. 77, n. 11, p. 1140-1143, 1993.
PENROSE, L. J.; KOFFMANN, W.; NICHOLLS, M. R. Field occurrence of vinclozolin resistance in Monilinia fructicola. Plant Pathology, London, v. 34, n. 2, p. 228234, 1985.

PEREIRA, F. M.; COUTINHO, E. L. M.; OLIVEIRA, F. Z. de. Importância da adubação na qualidade das frutas de clima temperado. In: SÁ, M. E. de; BUZZETI, S. Importância da adubação na qualidade dos produtos agrícolas. São Paulo: Ícone, 1994. p. 161-175.

RITCHIE, D. F. Effect of dichloran, iprodione, procymidone, and vinclozolin on the mycelial growth, sporulation, and isolation of resistant strains of Monilinia fructicola. Plant Disease, Saint Paul, v. 66, n. 6, p. 484-486, 1982.

SÔNEGO, O. R.; GARRIDO, L. da R.; CZERMAINSKI, A. B. C. Avaliação do fosfito de potássio no controle do míldio da videira. Bento Gonçalves: Embrapa, 2003. 14 p. (Boletim de Pesquisa e Desenvolvimento, 11).

WADT, L.; NOGUEIRA, E. M. de C.; CORRENTE, J. E. Controle químico da podridão parda (Monilinia fructicola) em nectarina (Prunus persica var. nucipersica). Arquivos do Instituto Biológico, São Paulo, v. 66, n. 1, p. 9-14, 1999. 